\title{
Sky Radiation Measurement and Corrections
}

\author{
N. Robinson and L. Stoch \\ Technion-Israel Institute of Technology, Haifa, Israel \\ (Manuscript received 10 December 1962, in revised form 27 May 1963) \\ ABSTRACT

\begin{abstract}
This paper presents data for constructing a shading device for a sky radiation recording station in any latitude up to $65 \mathrm{deg}$. In addition, tables are prepared for correcting the recorded radiation in order to obtain a standardized value of the sky radiation.
\end{abstract}

\section{Introduction}

The sky radiation is the part of the solar radiation caused by the scattering of the pure atmosphere and its various constituents.

The sky radiation $D_{\gamma}$ as a component of the solar radiation is defined as the amount of energy incoming on a horizontal surface from the total hemisphere. It is usually measured in cal $\mathrm{cm}^{-2} \mathrm{~min}^{-1}$. In order to measure its value the direct sun radiation must be cut off from the receiver of the measuring instrument by some shading device. There are two types of shadings; one rotary, driven by an equatorial mounting casting a shadow on the measuring instrument at any time of day and year; the other, fixed and adaptable to the appropriate astronomical and geographical conditions.

The sky radiation can, under certain conditions, be very appreciable. Exact measurement of this radiation component is hampered by lack of standardization: the sensitive elements in the various pyranometers are of different dimensions as are the glass globes protecting them. In addition to this, shadings of different dimensions are used for the same kind of pyranometers and vice-versa. To further complicate the situation, the shadings are mounted at varying distances from the receiver. (For details see Robinson, 1964.)

The Weather Bureau in Washington introduced a shading ring ${ }^{1}$ which the first author subsequently improved (1955). Other papers on the problem of shadings include those of Drummond (1956), Blackwell ${ }^{2}$ and the IGY manual (1958), where an additional bibliography is given.

${ }^{1}$ Hand, I. F., 1946: U. S. Weather Bureau, Washington (November), p. 55 .

${ }^{2}$ Blackwell, M. J., 1954: Five years continuous recording of total and diffuse solar radiation at Kew Observatory. Air Ministry Meteor. Res. Com., M.R.P.S.C/III/179, London.
Formulae for the corrections for any time of the year, caused by the obstruction of part of the sky by the shading frame according to Blackwell, are derived here. Correction tables are compiled and are also represented graphically. With the aid of these tables, the sky radiation, as recorded by the apparatus described in Section 2 , should be corrected, thereby standardizing the measured radiation and permitting unbiased comparison of the sky radiation for different regions. ${ }^{3}$

\section{The shading frame}

In Fig. 1, a general view of an Eppley pyranometer with a shading frame is seen. ${ }^{2}$ The frames are parts of a sphere; thus a constant distance between any part of the frame and the center of the receiver is secured throughout the year. The frames are attached to a frame support consisting of a horizontal and vertical member, each a circle of radius 20 inches, with legs of suitable height at the points N.W. S. E.

In order to avoid interreflection by the bulb which may cause errors in the readings, the minimum width of a frame must be at least equal to the diameter of the glass bulb: 3 inches in the case of an Epply pyranometer. One inch more is added in order to render the shading frame usuable for several days at least, yet to keep the required number of frames reasonably small. For example, this additional inch enables the same frame to be used for one week at the equinox when the daily change in the sun's declination $(\delta)$ is greatest.

This proposed width corresponds to a change of $2^{\circ} .8$ in $\delta$, which calls for 8.5 frames, i.e., one frame for the equinox and eight additional ones. The same frames may be used for symmetrical north and south declinations of

${ }^{3}$ Tables and diagrams with explanations and instructions of construction can be obtained from the first author. 


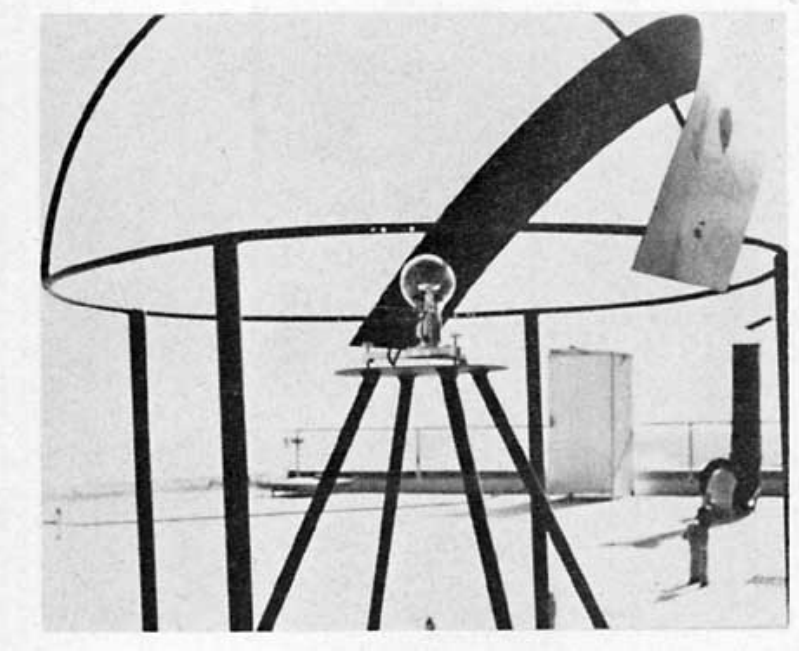

FIG. 1. General view of Eppley pyranometer with shading frame.

the sun by preparing two pairs of connecting points on each frame, in accordance with the sun's declination being northerly or southerly. To allow for a safety margin of 1-2 days, the width finally adopted for the shading frames is 4.2 inches.

\section{The correction data}

The symbols to be used in the following are:

$b=$ width of the shading frame

$b^{\prime}=$ width of the vertical member of the support

$r=$ radius of the sphere embodied by the shading frames

$\delta=$ declination of the sun

$t=$ hour angle of the sun

$t_{0}=$ hour angle of the sun at sunrise or sunset

$z=$ zenith distance

$\varphi=$ latitude of the station

$C=$ radius of disc required to cut out direct solar radiation

$D=$ the sky radiation.

The sky radiation measured by the pyranometer must be corrected for two cases:

1) for each individual frame

2) for the vertical member of the support.

To correct for these two, the measured radiation must be increased. The correction for (1) is divided into two parts: one-allowing for the whole frame to be added to the measured radiation; the other part-deducted from the first part to allow for the disc of suitable diameter required to obstruct the direct solar radiation at any instant. The correction for (2) is constant for any time of the year.

The formulae for the corrections are as follows.

\section{a) Formula for error $\Delta_{1} E_{D}$ caused by shading frame (con-} stant for each frame)

The radiation energy of the sky for the whole hemisphere is $E_{D}=\pi D$. The area of an element of a shadow frame is $b r \cos \delta d t$.

The solid angle is

$$
\left(b r / r^{2}\right) \cos \delta d t=(b / r) \cos \delta d t
$$

The energy of the element on the horizontal plane receiver is

$$
D \cos z(b / r) \cos \delta d t .
$$

The energy for the shading frame is

$$
\begin{aligned}
\Delta E_{D} & =D(b / r) \cos \delta \int_{-t_{0}}^{+t_{0}} \cos z d t \\
& =\underset{r}{2 D-\cos \delta\left(t_{0} \sin \varphi \sin \delta+\cos \varphi \cos \delta \sin t_{0}\right) .}
\end{aligned}
$$

This formula is almost identical with Drummond's (1956). The only difference being in the $\cos \delta$ term before the bracket which is $\cos ^{3} \delta$ for Drummond's device. This is because the distance from the shading frame to the receiving element and the effective width of the shading frame vary according to $\cos \delta$ each in Drummond's device, while in the device here described these two factors remain constant.

\section{b) Formula for the constant error $\Delta_{2} E_{D}$ caused by the vertical member of the support}

The energy for an element in the vertical plane is $\left.D \cos z\left(d z b^{\prime}\right) / r\right)$.

The energy

$$
\Delta_{2} E_{D}=D \frac{b^{\prime}}{r}-2 \int_{0}^{\pi / 2} \cos z d z=\frac{2 D b^{\prime}}{r} .
$$

The value of $\Delta_{2} E_{D} / E_{D}$ (assuming $b^{\prime}=0.25$ inch) is 0.008 .

The factor $1 /\left[1-\left(\Delta_{1} E_{D}+\Delta_{2} E_{D}\right) / E_{D}\right]$ to be multiplied by the measured energy in order to correct for the combined effect of $\Delta_{1} E_{D}$ and $\Delta_{2} E_{D}$ is given in tables for all 17 shadow frames for latitudes $0^{\circ}, 10^{\circ} \cdots 60^{\circ}$. For intermediate latitudes, the value of the factor may be found by interpolation.

The factor is also represented graphically to show how it varies for different values of $\varphi$ and $\delta$.

c) Formula for the variable factor $\Delta_{3} E_{D}$ allowing for the disc of the rotary type shading device

The energy amount at the receiving element at any instant is $\Delta_{3} E_{D}=\left(C^{2} / r^{2}\right) D \cos z$ from which $\Delta_{3} E_{D} / E_{D}$ $=\left(C^{2} / r^{2}\right)(\sin \delta \sin \varphi+\cos \delta \cos \varphi \cos t)$ where $C$ is the radius of the disc (1.5 inches). 
This factor varies in three dimensions $\delta, \varphi$, and $t$, but it is very small and is best shown graphically from where intermediate values may be found by interpolation. The complete correction factor should be

$$
1 /\left[1-\left(\Delta_{1} E_{D}+\Delta_{2} E_{D}-\Delta_{3} E_{D}\right) / E_{D}\right]
$$

but as $\Delta_{3} E_{D}$ is so small compared with $\Delta_{1} E_{D}$, the following approximation

1

$$
\begin{aligned}
& 1-\left(\Delta_{1} E_{\mathrm{D}}+\Delta_{2} E_{\mathrm{D}}-\Delta_{3} E_{\mathrm{D}}\right) / E_{D} \\
& \simeq \frac{1}{1-\left(\Delta_{1} E_{D}+\Delta_{2} E_{D}\right) / E_{D}}-\frac{\Delta_{3} E_{D}}{E_{D}}
\end{aligned}
$$

is sufficiently accurate and permits tabulation of the correction data. Thus, the factor $\Delta_{3} E_{D} / E_{D}$ may be deducted from $1 /\left[1-\left(\Delta_{1} E_{D}+\Delta_{2} E_{D}\right) / E_{D}\right]$ in order to find the complete correction factor. However, for most purposes, the variable factor $\Delta_{3} E_{D} / E_{D}$ may be neglected as it is so small.

All corrections and formulae are correct only in case of an isotropic sky, which assumption is not always correct. (See Robinson, 1964.)

\section{Comparison with other methods}

Comparing the apparatus and corrections here de scribed with one of the accepted methods, i.e., that of Drummond (1956), we find that:

a) The adjustable ring system (Drummond) may be mass produced for all latitudes while our frame system may be mass produced for zones varying $10^{\circ}$ in latitude. b) The adjustable ring system requires daily adjustment to keep up with the change in the sun's declination.

The frame system needs attention from once weekly (equinoxes) to once in two months (solstices).

c) The correction for the shading frame is smaller than for the adjustable ring system on account of the relatively large radius, 20 inches as against 12 inches, despite the increased width, 4.2 inches against 2.0 inches.

\section{Conclusion}

Solar stations embarking on sky radiation recording program, should find their work considerably reduced by means of the data presented in this paper for preparing and using a shading device which is relatively simple to construct and easy to maintain.

The tables of corrections to the recorded radiation energy permit direct standardization of the sky radiation, avoiding complicated formulae and involved procedures. This added facility should contribute to unbiased comparison of the sky radiation energy of different seasons and for different regions.

\section{REFERENCES}

Drummond, A. J., 1956: On the measurement of sky radiation. Arch. Meteor. Geophys. Bioklim., 7, 413-436.

Radiation Commission of I. A. M., 1958: Manual IGY. London, New York, Pergamon Press.

Robinson, N., 1955: An occulting device for shading the pyrheliometer from the direct radiation of the sun. Bull. Amer. meteor. Soc., 36, 32-34.

--_, 1964: Solar radiation. Amsterdam, Princeton, London Elsevier Publishing Co., $450 \mathrm{pp}$. 